\title{
Contaminants apportés aux matrices biolo- giques dans l'analyse des métaux et des élé- ments-trace au cours de la surveillance des travailleurs exposés en médecine du travail
}

\section{Products contaminating biological fluids in the analysis of trace-elements during monitoring of exposed workers in industrial medicine}

Didier OLICHON*(1), Olivier GUILLARD ${ }^{(2)}$, Alain PINEAU ${ }^{(3)}$

(1) Laboratoire PASTEUR CERBA - 95066 CERGY-PONTOISE Cedex 9

(2) Laboratoire de Biochimie et Toxicologie, Hôpital Jean Bernard - 86021 POITIERS Cedex (3) Laboratoire de Toxicologie, Faculté de Pharmacie - 44035 NANTES Cedex

* Auteur à qui adresser la correspondance : Didier OLICHON, Laboratoire PASTEUR CERBA 95066 CERGY-PONTOISE Cedex 9 - Tél : 0134402131 - Fax : 0134402159

(Reçu le 10 mai 2001 ; accepté le 20 juin 2001)

\section{RÉSUMÉ}

Dans le cadre de la surveillance des travailleurs exposés aux métaux et aux éléments-trace, très souvent le biologiste n'est pas maître du prélèvement et reçoit donc des échantillons sanguins et/ou urinaires dont la collection a été effectuée en dehors de son laboratoire avec un délai parfois ignoré du laboratoire d'analyse. Il est capital tant pour le biologiste que pour l'information des préleveurs, de connaitre les risques de contaminations des échantillons biologiques. Toutes les étapes concernant l'acte du prélèvement tant en matière de choix des matrices, du matériel utilisé et des modalités de recueil doivent être entourées de précautions nombreuses. Au niveau du laboratoire également les étapes pré-analytiques et analytiques doivent être rigoureuses afin

\section{SUMMARY}

As part of his work, the biologist must supervise the workers exposed to metals and trace-elements. He is not the one who sampled; he receives blood and/or urinary samples from outside and sometimes long after they have been collected.It is of main importance for the biologist and the sampler to know the contamination risks of the biologic blood or urinary sampling. Every steps regarding the sampling, the choice of the matrix, the equipment used and how it is sampled must be carefully surrounded. In the laboratory too, the pre and analytic steps must be strict and the contamination risks linked to the building, the treatment and the conservation of the samples must not be ignored. The knowledge of all these risks and consequently the precautions taken all along the 
de ne pas méconnaître les risques de contaminations liées aux locaux, au traitement et à la préservation des échantillons. La connaissance de l'ensemble de ces risques et par conséquence les précautions prises tout au long des étapes de la vie d'un échantillon biologique concourent à donner au prescripteur une garantie de la qualité des examens réalisés pour la surveillance des personnels exposés.

\section{MOTS-CLÉS}

Contamination, métaux, prélèvements, sang, urines.

\section{Introduction}

Certains travailleurs au cours de leur activité professionnelle sont exposés à des degrés variables selon les métiers aux dérivés inorganiques et organo-métalliques.

La prévention des risques auxquels ces personnels sont exposés nécessite d'une part la surveillance des atmosphères de travail par la mesure des substances dangereuses dans l'air inhalé et d'autre part. la surveillance biologique par la mesure des indicateurs biologiques d'exposition. Il peut s'agir des expositions liées aux éléments-trace et aux métaux lourds eux mêmes, mais également des modifications biochimiques provoquées par ces éléments ; c'est le cas de la protoporphyrinezinc ou de la $\beta 2$ microglobuline analysées dans le sang total pour le plomb et pour le cadmium respectivement. Actuellement les éléments-trace et les métaux lourds le plus souvent analysés dans les laboratoires sur des échantillons biologiques sont : l'aluminium, l'arsenic, le chrome, le manganèse, le mercure, le cadmium et le plomb.

Les concentrations biologiques de ces éléments sont en général faibles ; aussi, un certain nombre de règles concernant le prélèvement ne doivent pas être sousestimées afin de ne pas contaminer l'échantillon biologique. C'est le cas des métaux par l'apport supplémentaire de ces éléments au moment du prélèvement (certains entrent dans la composition des matériels de prélèvement comme les aiguilles par exemple ou dans celle de solutions anticoagulantes). De plus, ils sont présents dans l'air ambiant (aluminium, chrome, nickel et manganèse).

En effet, il existe très souvent une sous-estimation du risque de contamination ou de disparition de ces éléments par l'acte de prélèvement et de conservation des échantillons mais aussi une sous-estimation par les fabricants de matériels des risques de contamination de leur produit pour le dosage des éléments-trace et des métaux lourds.

Les différentes étapes doivent être entourées de précautions visant à maintenir l'intégrité du prélèvement : il est nécessaire de connaître la fiabilité du spécimen, de steps of a biologic sample life, guarantees the practitioner of the excellence of the analysis performed for exposure workers.

\section{KEY-WORDS}

Contamination, metals, collection, blood, urine.

savoir quelle matrice doit être prélevée et dans quelles conditions (dispositifs, moment opportun), et de respecter les conditions d'acheminement et de conservation adéquates.

\section{Les prélèvements}

\section{- Le choix des matrices}

Le choix du milieu biologique analysé pour la surveillance d'un élément dépend du métabolisme et du devenir de cet élément dans l'organisme mais aussi des facilités de dosage en regard des taux rencontrés ainsi que de l'existence de valeurs de référence pour permettre une bonne interprétation des résultats. Ainsi le plomb est dosé dans le sang total et son dosage dans les urines n'est pas utile sauf pour évaluer les concentrations obtenues après une thérapeutique chélatrice. L'intoxication par l'aluminium chez les sujets insuffisants rénaux hémodialysés est suivie par le dosage sérique ou plasmatique (patients traités en général par l'hydroxyde d'aluminium pour les hyperphosphorémies). Cependant, dans le cas de l'exposition professionnelle et notamment dans les usines de production de l'aluminium, la surveillance serait meilleure sur les urines dont la concentration semble refléter une exposition récente. En revanche, le risque de pollution de l'échantillon urinaire est considérable car l'aluminium est très ubiquitaire et d'importantes précautions doivent être prises pour ces recueils.

Les matrices utilisées en routine seront : le sang total, le sérum ou le plasma et la miction urinaire (Tableau I).

Malgré le tropisme de ces éléments pour certains organes ou tissus, les autres milieux pouvant être utilisés pour le dosage de ces éléments tels que les tissus, les cheveux, les ongles, la salive, le lait, etc.... ne sont pas d'usage dans l'exploration de routine concernant la surveillance professionnelle.

Le prélèvement de sang est relativement peu contaminant s'il est effectué selon des règles précises comparativement au recueil urinaire. Le sang total est préféré quand l'élément s'accumule dans les globules rouges (plomb, manganèse) ou lorsque la répartition intracel- 
Tableau I :

\begin{tabular}{|l|c|c|c|c|c|}
\hline & Sang & Sérum & Urines & $\begin{array}{c}\text { Moment du } \\
\text { prélèvement }\end{array}$ & $\begin{array}{c}\text { Valeurs population } \\
\text { générale }\end{array}$ \\
\hline Plomb & $\mathrm{X}$ & & & Début de poste & $<80 \mu \mathrm{g} / \mathrm{l}$ \\
\hline Manganèse & $\mathrm{X}$ & & & Fin de poste & $<10 \mu \mathrm{g} / \mathrm{l}$ \\
\hline Aluminium & & $\mathrm{X}$ & & $\begin{array}{c}\text { Fin de semaine } \\
\text { Fin de poste }\end{array}$ & $<10 \mu \mathrm{g} / \mathrm{l}$ \\
\hline Cadmium & $\mathrm{X}$ & & & Indifférent & $<5 \mu \mathrm{g} / \mathrm{l}$ (fumeur) \\
& & & $\mathrm{X}$ & Indifférent & $<2 \mu \mathrm{g} / \mathrm{l}$ (non-fumeur) \\
\hline & $\mathrm{X}$ & & & $\begin{array}{c}\text { Fin de semaine } \\
\text { Fin de poste }\end{array}$ & $<5 \mu \mathrm{g} / \mathrm{l}$ \\
\hline Mercure & & $\mathrm{X}$ & $\begin{array}{c}\text { Après au moins } 6 \text { mois } \\
\text { d'exposition } \\
\text { en début de poste }\end{array}$ & $<5 \mu \mathrm{g} / \mathrm{g}$ créatinine \\
\hline Arsenic & & & $\mathrm{X}$ & $\begin{array}{c}\text { Fin de poste (ou le matin } \\
\text { du jour suivant l'exposition) }\end{array}$ & $<10 \mu \mathrm{g} / \mathrm{g}$ créatinine \\
\hline Chrome & & $\mathrm{X}$ & $\begin{array}{c}\text { Fin de semaine au début } \\
\text { et à la fin de poste }\end{array}$ & $<5 \mu \mathrm{g} / \mathrm{g}$ créatinine \\
\hline
\end{tabular}

lulaire est équivalente à la répartition plasmatique (cadmium, mercure). Le choix du milieu urinaire seul est préféré quand l'élément est éliminé principalement par voie rénale et que le dosage sérique ou sanguin n'apporte pas d'information utile à la surveillance de l'exposition professionnelle. Les résultats des dosages urinaires sont exprimés en rapportant le résultat à l'élimination de la créatinine. Cependant les données obtenues ne doivent être prises en considération que pour des taux de créatinine au moins égales à $0.3 \mathrm{~g} / \mathrm{l}$, significatif d'une filtration rénale normale et d'une urine non diluée.

\section{- Les prélèvements sanguins}

Les systèmes de prélèvement de sang sous-vide sont généralement préférés pour des raisons de praticabilité et d'hygiène. Les réserves importantes sur l'utilisation de ces tubes (1) ne sont plus d'actualité en raison des efforts importants effectués par les fabricants sur la qualité de leurs produits d'une manière générale mais aussi de l'élaboration de produits directement adaptés à l'analyse des éléments-trace. Les tubes BD VacutainerTM (BectonDickinson) stériles en verre (tubes secs siliconés pour les prélèvements sériques ou les tubes avec héparine) conviennent pour la plupart des dosages des éléments de la surveillance professionnelle. Il en est de même avec les tubes S-Monovette ${ }^{\mathrm{TM}}$ SARSTEDT pour la recherche de traces de métaux plus particulièrement adaptés à l'analyse précise d'une douzaine d'éléments-trace.
Le recueil pour un dosage sur le sérum ne devra pas se faire sur tube avec gel séparateur ni tube contenant des billes.

Les prélèvements par seringue en verre ou plastique ou le recueil du sang à l'aide d'un cathéter en Téflon ${ }^{\mathrm{TM}}$ (système ouvert) sont potentiellement à risque de contamination par l'air ambiant de la salle de prélèvement ; il est à noter également que les seringues en plastique peuvent contaminer par l'extrémité caoutchoutée du piston. Les seringues en verre non jetables ne doivent pas être utilisées.

Les aiguilles sont composées d'alliages métalliques divers pouvant contenir entre autre de l'aluminium, du chrome et du manganèse. Certaines aiguilles siliconées sont utilisables sans précaution particulière. En fait, un recueil minimisant les apports exogènes contaminant par ces aiguilles pourrait consister à n'utiliser pour le dosage des éléments métalliques que le second tube de prélèvement en rejetant les premiers millilitres de sang. Le recueil pour le dosage sur sang total doit prendre en considération la qualité des solutions anticoagulantes utilisées. Certaines héparines peuvent contenir des éléments contaminants de même que les bouchons en caoutchouc dont la fabrication a utilisé des catalyseurs métalliques (zinc et manganèse).

La désinfection de la peau avant la prise de sang devra proscrire la solution de Dakin pour l'analyse du manganèse et tout désinfectant à base de mercure en cas de 
dosage de cet élément. L'alcool, les compresses peuvent aussi être des vecteurs de contamination. Le recueil urinaire doit se faire au moment adapté en regard de l'élimination de l'élément. Il est recommandé de doser l'arsenic urinaire en fin de poste et fin de semaine de travail et si le recueil est fait sur les urines du matin du jour suivant l'exposition (après 2 jours d'exposition au moins) ; ce système de recueil permet de limiter les risques de contamination externe par l'arsenic organique d'origine alimentaire (poissons, crustacés, eau de boisson).

\section{- Le recueil urinaire}

Le recueil urinaire peut se faire dans les toilettes d'un laboratoire, d'une infirmerie ou bien au domicile du patient. Quel que soit le lieu de prélèvement, des règles d'hygiène doivent être observées pendant ce recueil avec un lavage soigneux des mains en particulier et en minimisant le temps d'exposition du flacon ouvert à l'air ambiant. Les prélèvements effectués en fin de journée de travail qui visent à apprécier la dose de toxique absorbé par l'organisme doivent être entourés de précautions particulières visant à éliminer tout risque de majoration du taux par une contamination externe par les vêtements de travail, les particules pouvant tomber des cheveux, les mains non lavées ou la cigarette aux lèvres...

Les flacons en polyéthylène ou en polypropylène utilisés seront neufs et stockés à l'abri des poussières ou des courants d'air avec leur bouchon en place correctement vissé. Certains auteurs recommandent le lavage préalable des flacons par une solution d'acide chlorhydrique à $10 \%$ suivi de plusieurs rinçages à l'eau bidistillée $(2,3)$. Il faudra éviter l'utilisation de conservateurs tels que les antiseptiques mercuriels (Merseptyl, Mercryl, Merfène) pour l'analyse du mercure ; les urines peuvent ensuite être acidifiées afin d'obtenir un $\mathrm{pH}$ à 2 avec un acide type suprapur dont la pureté aura été vérifiée auprès du fournisseur.

\section{- Renseignements concernant le patient.}

Un interrogatoire peut être utile à l'interprétation des résultats des dosages en raison des habitudes alimentaires ou comportementales des patients.

L'alimentation apporte de l'arsenic sous forme de composés organiques en quantité non négligeable dans les aliments à base d'organismes marins et la méthode utilisée pour le dosage de l'arsenic urinaire doit en tenir compte. De même, le thé apporte des traces d'aluminium. Le tabac, les abats et les coquillages sont de grands pourvoyeurs de cadmium. L'inhalation de la fumée d'une cigarette augmente le taux de cadmium sanguin de $1,6 \%$.

Le mercure est apporté en quantité non négligeable par les produits de la mer : poissons et crustacés.

Le chrome est aussi rencontré dans la bière et le tabac. Pour le plomb, il faut tenir compte d'une possibilité de contamination en dehors d'une exposition professionnelle qui peut se surajouter avec l'eau de boisson dans certaines régions et surtout, l'utilisation de certains ustensiles de cuisine ou bien de poteries telles que celles utilisées dans les tajines (habitudes et utilisations de conteneurs traditionnels).

\section{Étape pré-analytique - Centrifugation et décantation}

Après coagulation, la séparation se fera avec le souci d'éviter tout risque de contamination par l'air ambiant aux différents moments telles que la centrifugation, la décantation et la séparation dans un tube à hémolyse en polypropylène ou en polyéthylène avec fermeture par des bouchons en plastique.

\section{- Conservation}

Lorsque le dosage est différé en raison de la réalisation de mesures regroupées, les échantillons doivent être conservés dans des conditions qui n'altéreront par leur intégrité. Les données de la littérature sont diverses en ce qui concerne la conservation des échantillons à $+4{ }^{\circ} \mathrm{C}$ et à $-20^{\circ} \mathrm{C}$. Ces variations sont la plupart du temps imputables aux phénomènes d'adsorption sur les parois du tube en plastique et ceci d'autant plus que les éléments ont une faible liaison aux protéines de transport spécifiques et non spécifiques du plasma.

Un inconvénient majeur des échantillons de sang total conservés à $+4{ }^{\circ} \mathrm{C}$ pendant quelques jours à plusieurs semaines est la formation d'agglomérats érythrocytaires diminuant l'homogénéité de l'échantillon et pouvant fausser le résultat. Il conviendra alors d'homogénéiser par agitation rotative lente et pendant un temps suffisamment long ces échantillons dans les instants précédant le dosage. Pour le plomb, l'héparine pure gênerait la stabilité de l'échantillon $(2,3)$.

En ce qui concerne les urines, si elles ne sont pas analysées rapidement, il conviendra d'acidifier au laboratoire le prélèvement avec l'acide nitrique (4). Le recueil et la conservation du mercure urinaire initialement recommandés sur des flacons en verre en raison de phénomènes d'adsorption sur les parois pourraient se faire sur flacons en polypropylène ou en polyéthylène (5).

Il est reconnu que l'aluminium en solution aqueuse et dans les urines s'adsorbe fortement sur les flacons. Ceci est utile également pour les conservations des standards de dosages et des gammes dans les solutions analytiques. 


\section{Partie analytique}

\section{- Locaux}

Des conditions locales de travail en atmosphère contrôlée (pressurisée et filtrée) sont idéales (6). Dans un local ou l'atmosphère n'est pas contrôlée, on évitera les pièces avec les portes et les fenêtres ouvertes ainsi que le brassage de l'air pendant les manipulations ainsi qu'une disposition des automates d'absorption atomique en dehors des arrivées d'air conditionné.

Par ordre décroissant d'importance en contamination des locaux, les éléments sont le chrome, le nickel, le manganèse, l'aluminium, le cadmium et le plomb.

Des précautions pour le dépoussiérage du local, le nettoyage des paillasses et du sol sont nécessaires en ayant connaissance des produits chimiques utilisés. Une grande rigueur doit être observée sur la propreté des surfaces de travail.

Les matériaux de construction des locaux sont à prendre en considération (structures en aluminium ou autres éléments).

Le personnel travaillant dans ces locaux, par ses cheveux, ses cosmétiques (rouge à lèvres contenant du zinc et de l'aluminium), la propreté de ses mains après avoir fumé (en dehors des locaux de travail), ses bijoux (bracelets, bagues) peut contribuer à des contaminations qui peuvent perturber les analyses.

Les gants en latex ne sont pas utilisables car ils contiennent des éléments (comme le zinc) biologiquement importants. Les gants en vinyle sont utilisables.

Les précautions seront d'éviter les va-et-vient et les courants d'air, de limiter l'entrée de la pollution atmosphérique en filtrant l'air, de séparer les automates de mesure et la préparation des échantillons, de tester les produits de nettoyage des surfaces de paillasse ou du sol.

D'une manière générale, le laboratoire doit être dépourvu de tout ce qui n'est pas indispensable à l'analyse et il est conseillé de ne pas agrémenter les conditions de travail par des plantes vertes!

\section{- Traitement des échantillons}

La manipulation des échantillons préalable à leur dosage (transvasements, dilutions, ajouts de réactifs) sont aussi des étapes potentiellement contaminantes si des précautions ne sont pas prises. Les pipettes automatiques, les embouts de pipettes, les tubes en polyéthylène et en polypropylène, les bouchons sont susceptibles d'être contaminants. En cas de doute, on pourra les tester pour vérifier l'absence de pollution et les laver par trempage dans de l'acide chlorhydrique à $10 \%$ pendant une nuit, suivi de plusieurs rinçages à l'eau bi-distillée. La conservation de ces matériels rincés pourra se faire à l'étuve ou dans des boites à l'abri de l'atmosphère du laboratoire.
Les réactifs utilisés comme les agents mouillants tels que le Triton ou bien les modificateurs de matrice devront être constitués de produits ultrapurs (7) et leur stockage devra s'effectuer dans des flacons de préférence en plastique dont la pureté sera vérifiée.

\section{Conclusion}

La réalisation des analyses en spectrométrie d'absorption atomique électrothermique nécessite non seulement l'acquisition des compétences techniques théoriques et l'utilisation d'un matériel fiable et bien entretenu mais aussi la connaissance de bonnes pratiques permettant de garantir l'exécution rigoureuse des modes opératoires de cette discipline. C'est ainsi que les précautions prises au cours des différentes étapes précédant le dosage sont indispensables afin d'éviter les contaminations extérieures pouvant souiller l'échantillon et conduire à des résultats erronés sans rapport avec l'efficacité des protections au poste de travail des personnels exposés aux métaux lourds et aux éléments-trace. La prise en compte des précautions énoncées au cours des étapes de prélèvement, pré-analytique et analytique pour éviter les contaminations doivent permettre de donner au prescripteur le meilleur résultat possible qui est le reflet des moyens mis en œuvre pour protéger individuellement et collectivement la population exposée au cours de son activité professionnelle.

\section{Références}

1. Helman E.Z., Wallick D.K., Reingold I.M. Vacutainer contamination in trace element studies. Clin. Chem. $1971 ; 17: 61-62$.

2. Pineau A., Guillard O., Chappuis P., Arnaud J., Zawislak $\mathrm{R}$. Sampling conditions for biological fluids for trace elements monitoring in hospital patients : a critical approach. Crit. Rev. Clin. Lab. Sci. 1993 ; 30(3) : 203-222.

3. Chappuis P., Pineau A., Guillard O., Arnaud J., Zawislak $R$. Conseils pratiques concernant le recueil des liquides biologiques pour l'analyse des éléments-trace. Ann. Biol. Clin. 1994 ; 52 : 103-109.

4. Poupon J. Prélèvement. In : Assurance de qualité de l'analyse des éléments-trace en biologie. Technique et Documentation - Lavoisier, 1997 : 71-101.

5. Boiteau H.L., Pineau A. Mercury. In : Mc Kenzie H.A., Smethe L.E., eds. Quantitative element of biological materials Elsevier, Amsterdam, 553-560.

6. Baruthio F., Ducros V. Contaminations - Pollutions. In : Assurance de qualité de l'analyse des éléments-trace en biologie. Technique et Documentation - Lavoisier, 1997 : 35-57.

7. Chappuis P. Généralités. In : Techniques d'analyse des oligoéléments chez l'homme. Technique et Documentation - Lavoisier, 1995 : 1-31. 